\title{
Low Power, High-Speed ADCs and Digital Circuits for SKA
}

\author{
T. Tauqeer^, J. Sexton, M. Mohiuddin, and M. Missous
}

School of Electrical and Electronic Engineering, University of Manchester, UK.

\begin{abstract}
The design of ultra low power $(<300 \mathrm{~mW})$, high-speed analogue to digital converter (ADC) is an essential element for the Square Kilometre Array (SKA). This paper describes the design and simulation of a low-power high-speed (4GS/s) analogue to digital converter (ADC) based on two designs of InP/InGaAs Single heterojunction bipolar transistor (SHBT) $\left(5 \times 5 \mu \mathrm{m}^{2}\right.$ and $1.5 \times 5 \mu \mathrm{m}^{2}$ emitter area device). The essential difference between these two devices was the process reliability (yield), and thus the overall unit cost. Both devices provided DC and RF performance characteristics ideally suited for the low-power IC design, with high current gain of 70-80. The high-frequency performances differ due to the device geometry with an $\mathrm{ft} / \mathrm{fmax}=78 \mathrm{GHz} / 38 \mathrm{GHz}$ for the $5 \times 5 \mu \mathrm{m}^{2}$ and $\mathrm{ft} / \mathrm{fmax}=91 \mathrm{GHz} / 83 \mathrm{GHz}$ for the $1.5 \times 5 \mu \mathrm{m}^{2}$ emitter area device.
\end{abstract}

\section{Introduction}

Logic gates that are based on current mode logic (CML) and emitter coupled logic (ECL) circuits are an ideal choice for applications with high clock rates Nah et al. (1993); Montgomery et al. (1991); Hafizi et al. (1992); Yinger et al. (1993). CMOS technology is limited in high precision applications, such as ADCs due to the stringent requirement of device matching. Also to achieve high-speed (fT $>100 \mathrm{GHz}$ ) CMOS requires deep sub-micron gates (90nm or less) where expensive phase shift masks are required. HBTs on other hand offers high power handling capability, high current drive capability, low 1/f noise characteristics, excellent threshold voltage control and high speed performance with low power dissipation for the digital circuits.

\section{Growth and Fabrication}

The technology was developed at the University of Manchester (UOM) and used molecular beam epitaxy (MBE) growth which relied upon two novel developments. Firstly stoichiometric conditions permitted growth at a fairly low temperature of $420 \mathrm{oC}$ while conserving extremely high quality materials. Secondly, dimeric Phosphorus was generated from a gallium Phosphide (GaP) decomposition source leading to excellent RF device properties. The epitaxial layer structure of SHBT is shown in Table 1 . The relaxed geometry devices of area $5 \times 5 \mu \mathrm{m}^{2}$ and $1.5 \times 5 \mu \mathrm{m}^{2}$ were fabricated using a triple mesa, wet etching process. Epitaxial layers were first etched using a non-selective Ortho-Phosphoric based etchant H3PO4:H202:H20 (3:1:50). This was followed by a short selective etch of $\mathrm{HCl}: \mathrm{H} 20$ (1:1) to expose the InGaAs base and sub-collector layers. Non-alloyed Ti/Au contacts to the emitter, base and sub-collector layers were then thermally evaporated to complete the devices.

\footnotetext{
^ TauseefTauqeer@gmail.com
}

Table 1: Epitaxial structure of SHBT sample

\begin{tabular}{|l|c|c|c|}
\hline Layer & \multicolumn{1}{|c|}{ Material } & \multicolumn{1}{|c|}{$\begin{array}{c}\text { Doping } \\
\left(\mathrm{cm}^{-3}\right)\end{array}$} & $\begin{array}{c}\text { Thickness } \\
(\AA)\end{array}$ \\
\hline Cap & $\mathrm{In}_{0.53} \mathrm{Ga}_{0.47} \mathrm{As}$ & $n=1 \times 10^{19}$ & 1350 \\
Emitter 1 & $\mathrm{In}_{0.53} \mathrm{Ga}_{0.47} \mathrm{As}$ & $n=1 \times 10^{19}$ & 1350 \\
Emitter 2 & $\mathrm{InP}$ & $n=1 \times 10^{17}$ & 400 \\
Spacer & $\operatorname{In}_{0.53} \mathrm{Ga}_{0.47} \mathrm{As}$ & - & 50 \\
Base & $\mathrm{In}_{0.53} \mathrm{Ga}_{0.47} \mathrm{As}$ & $p=1.5 \times 10^{19}$ & 650 \\
Collector & $\mathrm{In}_{0.53} \mathrm{Ga}_{0.47} \mathrm{As}$ & $n=1 \times 10^{16}$ & 6300 \\
Sub-Collector & $\mathrm{In}_{0.53} \mathrm{Ga}_{0.47} \mathrm{As}$ & $n=1 \times 10^{19}$ & 5000 \\
Buffer & $\operatorname{In}_{0.53} \mathrm{Ga}_{0.47} \mathrm{As}$ & - & 100 \\
\hline Substrate & \multicolumn{3}{|c|}{ Semi-Insulating InP } \\
\hline
\end{tabular}

\section{Device Modelling}

The measured common-emitter characteristics and cut-off frequency verssus the collector current of the two devices are shown in Figure 1 and Figure 2, respectively. As depicted in Figure 2, SHBT_1.5 $\times 5 \mu \mathrm{m}^{2}$, devices were the most suitable for the low-power high-speed applications because of demonstrating high frequency characteristics at relatively low values of collector current. However, SHBT $1.5 \times 5 \mu \mathrm{m}^{2}$ were not fabricated because of the poor yield ( $30 \%)$. On the other hand, SHBT $5 \times 5 \mu \mathrm{m}^{2}$ showed a good yield of over $90 \%$ and thus were favourable from fabrication point of view. The simulations were carried out for both of the samples and a comparison study is made.

Transistor parameters were extracted Maas et al. (1992); Lee et al. (2005) from the transistor for non-linear modelling in Agilent's ICCAP and Advanced Design System (ADS) software. This software includes the small-signal University of San Diego (UCSD) model (standard SPICE Gummel-Poon modified model) for the HBT UCSD (2000). The modelled and measured common emitter characteristics of SHBT $5 \times 5 \mu \mathrm{m}^{2}$ and SHBT_1.5 $\times 5 \mu \mathrm{m}^{2}$ are shown in Figure 3 and Figure 4, respectively. 


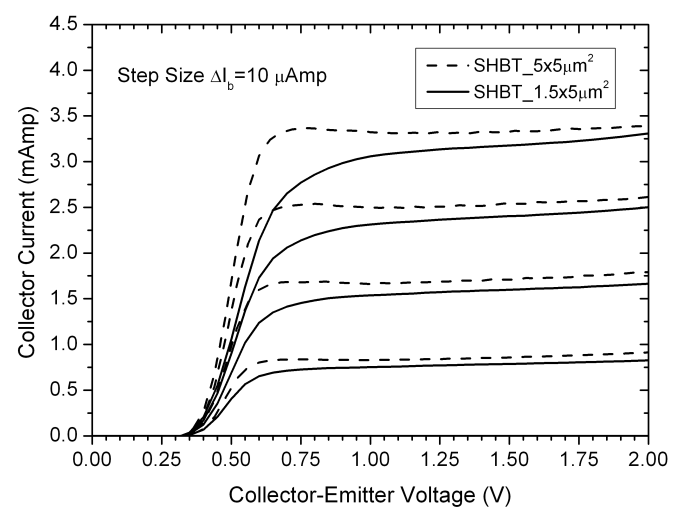

Fig. 1: IV Characteristics of two samples

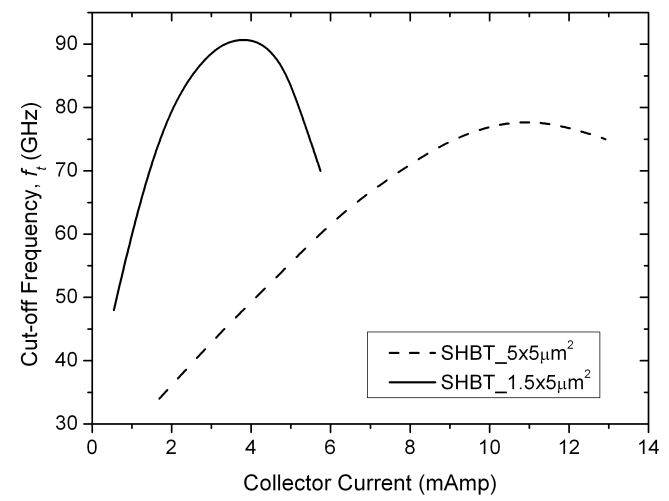

Fig. 2: Ic vs ft comparison of two samples

\section{Circuit Simulations}

\subsection{Comparator}

The most important building block of the flash ADC is the differential latching comparator (Figure 5). The speed of the latching comparator is determined mainly by its recovery time, $t_{r}$, which can be expressed Hotta et al. (1986) by

$$
t_{r}=C_{L} R_{L} \equiv C_{L} \frac{\Delta V_{0}}{I_{L}}
$$

where CL is the collector load capacitance, RL is the collector load resistance, IL is the tail current and VO is the output voltage swing.

The tail current for sample SHBT $5 \times 5 \mu \mathrm{m}^{2}$ is chosen to be $720 \mu \mathrm{A}$ with the recovery time of $166 \mathrm{ps}$ and which is well under the Nyquist frequency (500ps divided by 2 as level triggered clocking) for $1 \mathrm{GHz}$ input signal. The performance was acceptable for $333 \mathrm{MHz}$ input signal and up to $600 \mathrm{MHz}$ but there was a large slew rate that was comparable to $1 \mathrm{GHz}$ input signal and thus the circuit required further optimisation. The slew rate can be decreased by increasing the tail current and reducing the collector resistors. However, this exceeds significantly the power

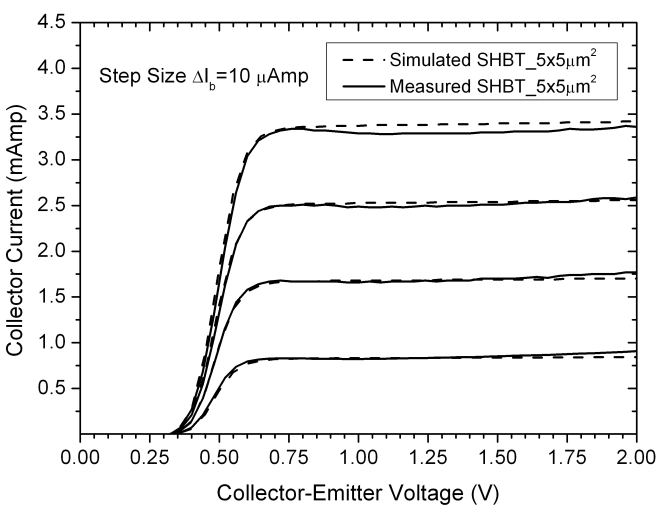

Fig. 3: Modelled and Measured SHBT_ $5 \times 5 \mu \mathrm{m}^{2}$

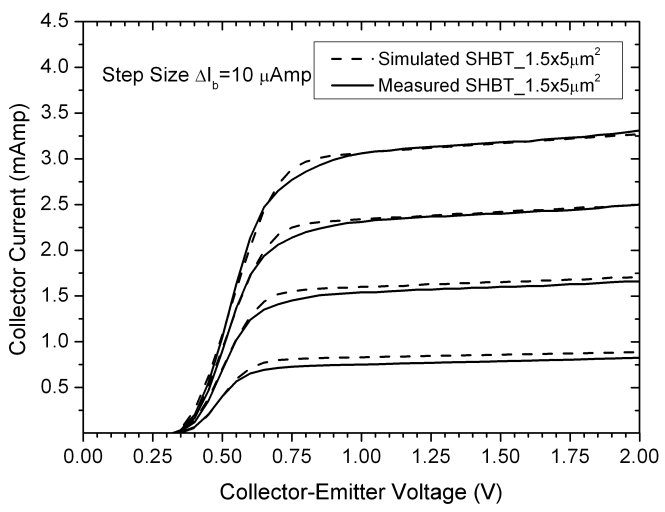

Fig. 4: Modelled and Measured SHBT_1.5 $\times 5 \mu \mathrm{m}^{2}$

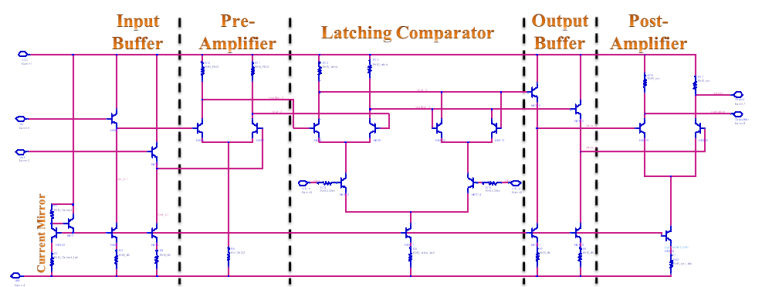

Fig. 5: Circuit Diagram of Differential Latching Comparator

limits - designing a 4-Bit ADC with power consumption less than $300 \mathrm{~mW}$.

The device was replaced by SHBT_ $1.5 \times 5 \mu \mathrm{m}^{2}$ which has improved ft and fmax at much lower collector current. The circuit was re-optimized (resistors and current mirror) and simulations were conducted on the latched comparator to decide on suitable load currents for this transistor at the clock frequency of $4 \mathrm{GHz}$. The higher clock rate helps in tracking the signal more accurately. The recovery time of $125 \mathrm{ps}$ is the upper limit for the latching comparator clocked at $4 \mathrm{GHz}$. To reduce the impact of meta-stable states, and improve sensitivity, a maximum 
recovery time of $60 \mathrm{ps}$ was chosen, setting the tail current to be $520 \mu \mathrm{A}$.

The simulated differential latched comparator results for two devices are shown in Figure 6. It can be easily observed that device $\mathrm{SHBT}_{-} 1.5 \times 5 \mu \mathrm{m}^{2}$ has given much better output characteristics as compared to SHBT $5 \times 5 \mu \mathrm{m}^{2}$ and thus chosen for 4-Bit Flash ADC simulation. The improved results were mainly due 4 times lesser CBC of SHBT $1.5 \times 5 \mu \mathrm{m}^{2}$ as compared to SHBT $5 \times 5 \mu \mathrm{m}^{2}$.

\subsection{Flash 4-Bit $A D C$}

The full 4-Bit flash ADC includes 15 comparators, 15 XORs, 32 diodes and resistor ladder producing a low-power consumption of $240 \mathrm{~mW}$ for $2 \mathrm{GHz}$ clock and $290 \mathrm{~mW}$ for a $4 \mathrm{GHz}$ clock.

\section{Conclusion}

The continuing research into the design an ultra low-power 4Bit A/D converter for use in the upcoming Square Kilometre Array (SKA) is presented in this paper. One design currently under investigation is the flash folding ADC, which has demonstrated a power consumption of $\sim 150 \mathrm{~mW}$. With further optimisation of the transistor epilayer design, an ultra-low power, $\mathrm{GHz}$ class ADC is possible using this technology.

\section{References}

M. Hafizi, J.F. Jensen, R.A. Metzger, W.E. Stanchina, D.B. Rensch, and Y.K. Allen, "39.5-GHz static frequency divider implemented in AlInAs/GaInAs HBT technology," Ieee Electron Device Letters, vol. 13, pp. 612-614, 1992.

M. Hotta, K. Maio, N. Yokozawa, T. Watanabe, and S. Ueda, "150-MW, 8-BIT VIDEO-FREQUENCY A/D CONVERTER.," IEEE Journal of Solid-State Circuits, vol. SC-21, pp. 318-323, 1986.

K. Lee, K. Choi, S.-H. Kook, D.-H. Cho, K.-W. Park, and B. Kim, "Direct parameter extraction of SiGe HBTs for the VBIC bipolar compact model," IEEE Transactions on Electron Devices, vol. 52, pp. 375-384, 2005.

S. A. Maas and D. Tait, "Parameter-extraction method for heterojunction bipolar transistors," IEEE Microwave and Guided Wave Letters, vol. 2, pp. 502-4, 1992.

R. K. Montgomery, P. R. Smith, F. Ren, T. R. Fullowan, C. R. Abernathy, R. F. Kopf, S. J. Pearton, J. Lothian, P. Wisk, and R. N. Nottenburg, "10 Gbit/s high sensitivity low error rate decision circuit implemented with C-doped AlGaAs/GaAs HBTs," Electronics Letters, vol. 27, pp. 976-8, 1991.

K. Nah, R. Philhower, H. Greub, and J. F. McDonald, "500 ps 328 register file implemented in GaAs/AlGaAs HBTs," San Jose, CA, USA, 1993, pp. 71-74.

"University of California San Diego (UCSD)," HBT MODELING, http: //hbt . ucsd. edu, 2000

S. Yinger, F. Lee, R. T. Huang, K. Schneider, E. Wang, K. Smith, M. Penugonda, S. Jacobs, and T. Carter, "HBT gate array for 5 GHz ASICs," San Jose, CA, USA, 1993, pp. 8790. 

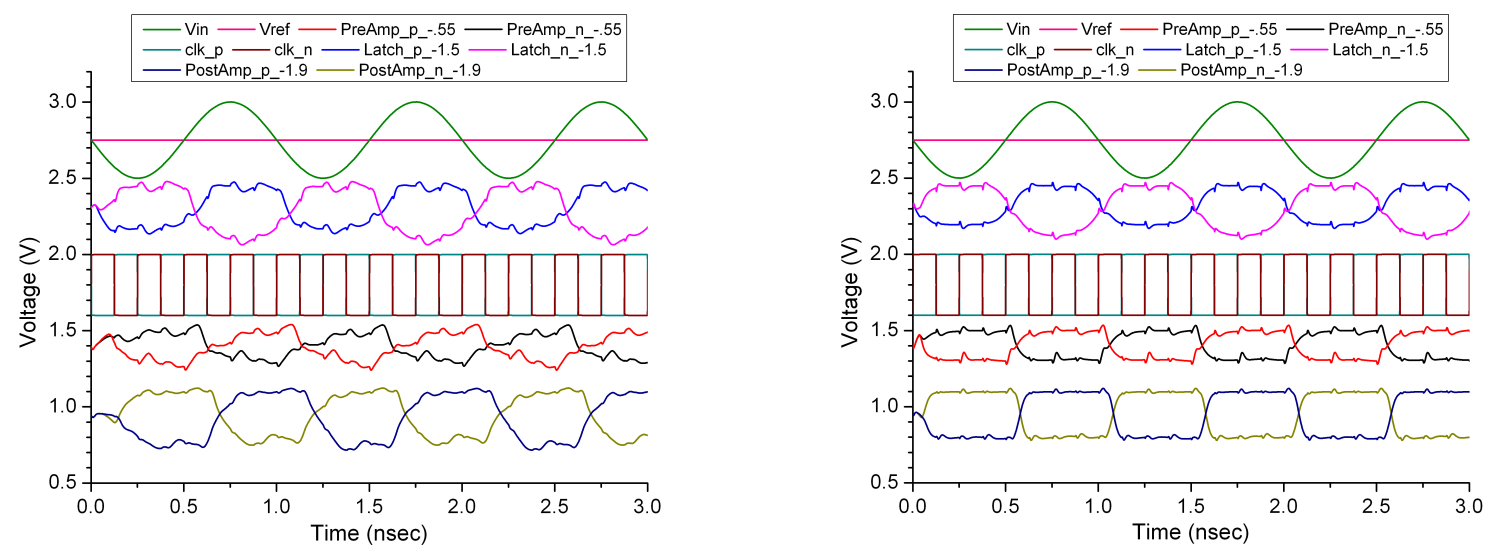

Fig. 6: Comparison of Simulation Results of Different Samples left: 4GHz Clock, 1GHz Input Signal SHBT_5 $\times 5 \mu \mathrm{m}^{2}$ right: $4 \mathrm{GHz}$ Clock, $1 \mathrm{GHz}$ Input Signal SHBT_1.5 $\times 5 \mu \mathrm{m}^{2}$ 